\title{
New Concepts in the Causes and Control of Mastitis
}

\author{
Lorraine M. Sordillo
}

Published online: 15 October 2011

(C) Springer Science+Business Media, LLC 2011

Mastitis is an inflammation of the mammary gland that is most often caused by bacterial infection and can affect all lactating mammals. Results from epidemiological studies suggest that up to a third of all lactating women will become ill with mastitis. Prompt diagnosis and treatment of mastitis in women is required to prevent lactation failure, recurrent mastitis, breast abscess, and even death in some situations [1]. Mastitis also is a significant health problem in food-producing animals such as dairy cattle. Results from the most recent National Animal Health Monitoring Survey indicate that mastitis continues to be the most prevalent disease affecting adult dairy cows in the United States [2]. Economic losses associated with mastitis will cost American dairy producers approximately $\$ 2$ billion in lost profits each year. Factors that impact the profitability of dairy production include decreased milk production and quality, treatment costs, replacement animal costs, and reduced ability to market dairy products. Even though there has been considerable research into the development of effective prevention and treatment strategies to control the disease, mastitis continues to be a significant issue in both human and veterinary medicine.

The outcome of microbial invasion of the mammary gland depends on the complex interaction between pathogens that cause disease, the host responses that are needed to eliminate the infectious agent, and the various risk factors that will influence pathogen virulence and mammary gland defense mechanisms. For example, the incidence and severity of mastitis is greatest during the periparturient period in both humans and dairy cows. During this time, the mammary gland experiences increased exposure to a plethora of mastitis-causing pathogens, but at the same

L. M. Sordillo $(\bowtie)$

College of Veterinary Medicine, Michigan State University,

East Lansing, MI 48824, USA

e-mail: sordillo@msu.edu time, important immunological defense of the host are compromised. A better understanding of important hostpathogen interactions that lead to the pathogenesis of mastitis will be needed in order to design effective mastitis control strategies. This special issue examines the available research concerning mammary gland responses to infection and the predisposing factors that are important to the development of mastitis. The latest advances into the causes and consequences of mastitis in humans and domestic food-producing animals will be discussed in detail.

Successful lactations are central to the reproductive performance of nearly all mammals. Optimal milk yield is dependent upon adequate proliferation and differentiation of mammary epithelial cells during the periparturient period and events that interfere with these processes can reduce the capacity to synthesize milk. Mastitis that occurs immediately before or after the onset of lactation impacts mammary function in several ways including disruption of alveolar cell integrity, induction of apoptosis, and interference with cellular differentiation. Using the ruminant mammary gland to illustrate these concepts, Akers and Nickerson examines the impact of mastitis on mammary gland structure and function with consideration of the relationship between epithelial cell proliferation and differentiation. Quantitative morphological studies have emphasized the importance for optimal differentiated alveolar epithelial cells to meet full milk production potential.

Mastitis can present as varying degrees of severity ranging from mild disease with no visible changes in the milk or mammary tissue to clinical disease with obvious changes to the mammary gland. Clinical symptoms of disease may include typical signs of inflammation including swelling, redness, and pain. The severity of mastitis may also progress to include systemic involvement such as fever, depression, loss of appetite and even death in some of the more severe cases. Inflammation of the mammary gland is an important host response to invading pathogens, 
but escalation of antimicrobial defenses can have a detrimental impact on host tissue and can disrupt and impair mammary gland function. Therefore, it is important that the inflammatory response be sufficiently rapid and robust during the early stages of infection to adequately eliminate the invading pathogen before significant damage to mammary tissue occurs. The review by Aitken, Corl and Sordillo explores in detail the current knowledge of critical host-pathogen interactions that occur during mastitis that can either result in the elimination of invading pathogens and restoration of immune homeostasis or lead to uncontrolled inflammatory responses that can disrupt normal mammary gland function. These authors also address risk factors that diminish the efficiency of innate and adaptive immune responses that may contribute to pathogenesis of severe mastitis. The review by Boehmer extends what is currently known about host responses to mastitis-causing pathogens by suggesting how proteomic analysis of healthy versus mastitic milk can offer utility in better characterizing mechanisms of disease and to uncover potentially novel targets for therapeutic intervention. The prospects of using proteomic analysis as a means to identify reliable inflammatory biomarkers were discussed in the context of early mastitis detection protocols and as a tool to screen for drug efficacy. As a complementary approach to proteomics, Loor and colleagues describe how the application of microarray technology can be used to study mastitis and provide an enormous amount of fundamental information on how the mammary gland adapts during intramammary infections. The use of high-throughput gene expression has been used extensively in recent years to dissect both the pathogen and mammary tissue adaptations during infection. These authors provide an intriguing discussion of how transcriptomics can have unique utility in a systems biology approach to better identify potential predisposing factors that influence the incidence and severity of mastitis. The concept of incorporating the biological complexities of the whole animal to uncover underlying links within and between tissues of affected animals have the potential to discover new therapeutic targets based on a holistic view of how the animals functions during mastitis pathogenesis.

Mastitis can be caused by a wide variety of organisms including gram-positive and gram-negative bacteria, mycoplasmas, and algae. As such, the response of the mammary gland to invading pathogens will vary based on the virulence of the etiological agent. The relative infectivity of the etiological agents also will be influenced greatly by the environment of both the host and the invading microbe. The review by Contreras and Rodriguez discuss the complex interaction of the host, microbes and environmental factors that influence the development of mastitis among different mammals. They provide a unique perspective of the comparative etiology and epidemiology of mastitis in humans and food-animal species by highlighting areas of similarity and diversity. The challenges for accurate etiological diagnosis are discussed in the context of both human and veterinary medicine. Zadoks and colleagues also evaluated the important contribution that molecular epidemiological studies have had to our current understanding of sources, transmission routes, and prognosis for many bovine mastitis pathogens with comparative relevance to human medicine. They provide an in-depth evaluation of how DNA-based characterization of mastitis-causing pathogens at the subspecies level has contributed to our understanding of how pathogens evolve to evade host defenses in addition to how identification of allelic profiles for housekeeping genes or virulence genes are useful diagnostic tools to assess pathogen persistence. In a complementary review, Holmes and Zadoks focus specifically on the epidemiology of methicillin resistant Staphylococcus aureus (MRSA) that are responsible for a relatively small number of human and bovine mastitis cases. The potential for farm animals to act as a reservoir for transmitting MRSA to other food-animals or humans is discussed based on the current literature. Collectively, both of these reviews highlights the strengths and weaknesses of using molecular biology tools to generate a deeper understanding of mastitis epidemiology for the diverse pathogen populations that can cause disease.

The significant impact that mastitis has on human and veterinary health has focused considerable research efforts to find more effective methods to treat and prevent disease. Currently, antibiotic treatment is an established component of mastitis control in both women and food-animal species. The diversity in eitiological agents, however, creates some difficulties for finding the most appropriate antimicrobial therapy to treat mastitis cases. The review of Barlow addresses the challenge of linking in vitro antimicrobial susceptibility testing as a predictor for mastitis treatment outcomes in dairy cattle based on the numerous field trials conducted over the past 25 years. The author comments on how antimicrobial susceptibility testing results can be used as a predictor of bacterial cure in the context of antimicrobial drug efficacy testing. Whereas antimicrobial therapies are commonly used to treatment mastitis, there is a concern for the potential development of antibiotic resistant strains or the potential contamination of the food-supply with antibiotic residues. For this reason, the prevention of intramammary infections through vaccination is a major goal within the mastitis research community. Attempts to develop efficacious mastitis vaccines, however, have met with variable results. The complexity and variation of pathogen virulence factors and a limited knowledge of adaptive immune responses of the mammary gland has been a significant hurtle to successful mastitis vaccine formulations. The review of Bharathan and Mullarky critically evaluates previous attempts to vaccinate against bovine mastitis. They also speculate on the prospects 
of future mastitis vaccine successes as our current understanding of mammary gland immune mechanisms expand. They emphasize the need to develop vaccine protocols that enhance mucosal cellular immunity and trafficking of memory $\mathrm{T}$ cells to the mammary gland as a strategy to develop effective immunization against specific mastitiscausing pathogens. The genetic background of an animal also can dictate disease susceptibility. The final paper by Pighetti and Elliot explores the potential of controlling mastitis through maker-assisted selection. Recent studies have examined the use of polymorphisms to better define the genes and chromosomal regions that contribute to bovine mastitis resistance. The prospects of using both genome wide association studies and candidate gene based studies to identify the best candidates for marker-assisted selection of mastitis resistant dairy cows is discussed in detail. The authors also provide valuable insights for the potential benefits of selecting for disease resistance as well as some of the obstacles that must be overcome for practical applications in the future.

This special issue on the causes and control of mastitis provides a compilation of in-depth reviews that emphasize the complex interaction between the immunological defenses of the mammary gland, diversity of microorganisms that cause infection, and the environment of both the host and pathogens that contribute to mastitis. Although considerable progress in treatment, prevention and diagnosis of mastitis has been made over the last decade, all of these reviews emphasize the need for additional research to achieve reductions in the incidence and severity of disease. The papers in this special issue provide a solid framework of how to address the future challenges of mastitis control in human and veterinary medicine.

\section{References}

1. Foxman B, D'Arcy H, Gillespie B, Bobo JK, Schwartz K. Lactation mastitis: occurrence and medical management among 946 breastfeeding women in the United States. Am J Epidemiol. 2002;155(2):103-14.

2. USDA. Dairy 2007, Part V: Changes in dairy cattle health and management proctices in the United States, 1996-2007. Fort Collins, CO: USDA:APHIS:VS, CEAH; 2009. 